\title{
Iterative Phase Difference Constraint Method for the Speckle Elimination of Fresnel Kinoforms
}

\author{
Toshinori Hora ${ }^{\mathrm{a}, *}$, Shiyuan Yang ${ }^{\mathrm{a}}$, Fenghui Yao ${ }^{\mathrm{b}}$ \\ ${ }^{a}$ Kyushu Institute of Techonology, Sensui-cho, Tobata-ku, Kitakyushu, Fukuoka, 804-8550, JAPAN \\ ${ }^{\mathrm{b}}$ Tennessee State University, 3500 John Merritt Boulevard Nashville TN, 37209-1561, USA \\ *Corresponding Author: hora@ boss.ecs.kyutech.ac.jp
}

\begin{abstract}
Speckle noise is a serious problem in the reconstruction of computer-generated holograms (CGHs). Especially for a kinoform (a phase-only CGHs), the speckles lead to a large reconstruction error and it is important to eliminate the speckles. Phase difference constraint method has been proposed as a method for speckle elimination ${ }^{(1)}$. However, simulation of phase difference constraint method is carried out only in the Fourier kinoform. But, considering the optical reconstruction, it preferred to use a Fresnel kinoform. Therefore, we carried out the simulation in Fresnel kinoform. We carried out the simulation in the three kinds of images "F", "I", "Y", and the speckle elimination has been confirmed in all images. Therefore, the phase difference constraint method is also useful in the Fresnel kinoform was confirmed.
\end{abstract}

Keywords: fresnel kinoform, speckle elimination.

\section{Introduction}

Speckle noise is a serious problem in the reconstruction of computer-generated holograms (CGHs). Especially for a kinoform (a phase-only CGHs), the speckles lead to a large reconstruction error and it is important to eliminate the speckles.

Phase difference constraint method has been proposed as a method for speckle elimination. However, simulation of phase difference constraint method is carried out only in the Fourier kinoform. But, in the Fourier kinoform, compared to the size of the hologram, the distance from hologram to reconstructed image must be very long. Considering the optical reconstruction, it preferred to use a Fresnel kinoform which can put close the distance between hologram and reconstructed image. So in this study, we carry out the simulation of phase difference constraint method in Fresnel kinoform.

\section{Fresnel kinoform}

As shown in Fig.1, we consider the reconstruct when there is kinoform in $\mathrm{z}=0$. When $f\left(x_{0}, y_{0}, 0\right)$ is the intensity of light in the point of $P\left(x_{0}, y_{0}, 0\right)$ on kinoform, we does approximate calculation as $g(x, y, z)$ is the intensity of light. $g(x, y, z)$ is the intensity of light on the point of $Q(x, y, z)$ in a plane perpendicular to the z-axis and is $r$ distant from the point of $P\left(x_{0}, y_{0}, 0\right)$.

$$
\begin{aligned}
& g(x, y, z)=\frac{1}{j \lambda} \iint f\left(x_{0}, y_{0}, 0\right) \frac{\exp (j k r)}{r \cos \varphi} d x_{0} d y_{0} \\
& r=\sqrt{z^{2}+\left(x-x_{0}\right)^{2}+\left(y-y_{0}\right)^{2}}
\end{aligned}
$$

$\varphi$ is the angle between the line $P Q$ and the $z$-axis. If the point of $Q$ is located in the $z$-axis near and $z$ is a large value adequate for the kinoform, $\cos \varphi$ equals 1 and denominator's $r$ equals $z$ from paraxial approximation. Therefore, Eq. (2) is possible to be approximated to Eq. (3).

$$
g(x, y, z)=\frac{1}{j \lambda} \iint f\left(x_{0}, y_{0}, 0\right) \exp (j k r) d x_{0} d y_{0}
$$

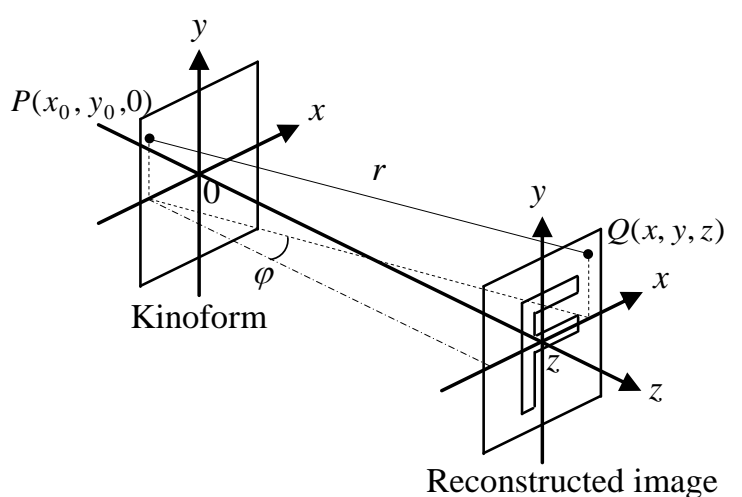

Fig.1. Reconstruction of the hologram 
Eq. (2) becomes Eq. (4) by binominal expression.

$$
\begin{aligned}
& r=z+\frac{\left(x-x_{0}\right)^{2}+\left(y-y_{0}\right)^{2}}{2 z} \\
&+\frac{\left\{\left(x-x_{0}\right)^{2}+\left(y-y_{0}\right)^{2}\right\}^{2}}{8 z^{3}}+\cdots
\end{aligned}
$$

It becomes Eq. (5) using the paraxial approximation.

$$
r \approx z+\frac{\left(x-x_{0}\right)^{2}+\left(y-y_{0}\right)^{2}}{2 z}
$$

Eq. (5) is substituted in Eq. (3).

$$
\begin{aligned}
& g(x, y, z)=\frac{\exp (j k z)}{j \lambda z} \iint f\left(x_{0}, y_{0}, 0\right) \\
& \quad \exp \left\{j k \frac{\left(x-x_{0}\right)^{2}+\left(y-y_{0}\right)^{2}}{2 z}\right\} d x_{0} d y_{0}
\end{aligned}
$$

It becomes Eq. (7) if sufficiently far from the hologram.

$$
\begin{aligned}
& g(x, y, z)=\frac{\exp (j k z)}{j \lambda z} \exp \frac{j k\left(x^{2}+y^{2}\right)}{2 z} \\
& \cdot \iint f\left(x_{0}, y_{0}, 0\right) \cdot \exp \left\{-j k \frac{x \cdot x_{0}+y \cdot y_{0}}{z}\right\} d x_{0} d y_{0}
\end{aligned}
$$

Eq. (7) can be expressed as Eq. (9) if we can regard $z$ as constant and perform a replacement of Eq. (8).

$$
\begin{aligned}
& u=\frac{x}{\lambda z}, v=\frac{y}{\lambda z} \\
& g(x, y) \propto \\
& \quad \iint f\left(x_{0}, y_{0}\right) \exp \left\{-j 2 \pi\left(u x_{0}+v y_{0}\right)\right\} d x_{0} d y_{0}
\end{aligned}
$$

We called the Fresnel kinoform, that is created by calculated from reconstructed image based on Eq. (6). Also, we called the Fourier kinoform, that is created by calculated from reconstructed image based on Eq. (9). Considering the optical reconstruction, it is desirable to use a Fresnel kinoform because a very long distance is necessary for to create the Fourier kinoform.

\section{Iterative phase difference constraint method for the speckle elimination}

\subsection{Outbreak mechanism of the speckle}

Speckle is a noise to occur in overall hologram technology because we use a coherent light such as laser light for the reconstruction and the record of a hologram. In

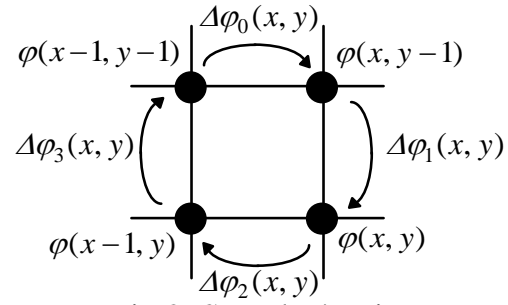

Fig.2. Sample 4 points computer-generated holograms, the speckles can be treated as the existing of isolated zero points among the sampled reconstructed distribution.

Fig. 2 shows the four adjacent sampled points in the reconstructed distribution. Denoting the phases as $\varphi(x, y)$, $\varphi(x-1, y), \varphi(x, y-1)$ and $\varphi(x-1, y-1)$, we define the phase difference between each the sampled points as follows:

$$
\begin{aligned}
& \Delta \varphi_{0}(x, y)=\varphi(x-1, y-1)-\varphi(x, y-1) \\
& \Delta \varphi_{1}(x, y)=\varphi(x, y-1)-\varphi(x, y) \\
& \Delta \varphi_{2}(x, y)=\varphi(x, y)-\varphi(x-1, y) \\
& \Delta \varphi_{3}(x, y)=\varphi(x-1, y)-\varphi(x-1, y-1)
\end{aligned}
$$

Supposing the phase period interval is $-\pi \leq \varphi<\pi$, the phase difference exists in the interval $-2 \pi<\Delta \varphi<2 \pi$. In this case, it is clear that

$$
\sum_{i=0}^{3} \Delta \varphi_{i}(x, y)=0
$$

However, when wrapping the phase difference into phase period interval $-\pi \leq \varphi<\pi$, Eq. (14) becomes

$$
\sum_{i=0}^{3} \Delta \tilde{\varphi}_{i}(x, y)=0, \pm 2 \pi,-4 \pi
$$

When the sum of phase differences becomes either value of $\pm 2 \pi,-4 \pi$, a speckle occurs. In other words, the speckle occurs for a phase difference existing across the range of $[-\pi, \pi]$. But, if the number of phase difference which exists in the range $(-2 \pi,-\pi)$ and the number of phase difference which exists in the range $[\pi, 2 \pi)$ are equal, the speckle does not occur because four points of sums to be adjacent by wrapping of phase difference do not have a change. Therefore we can define the condition that a speckle produces like the next expression.

The number of phase difference which exists in the range $(-2 \pi,-\pi)$

$\neq$ The number of phase difference

which exists in the range $[\pi, 2 \pi)$

\subsection{Iterative phase difference constraint method}

According the condition of Eq. (16), we limit the phase difference in range

$$
\begin{aligned}
& -2 \pi<\Delta \varphi^{\prime}(x, y) \leq-\frac{3}{2} \pi \\
& -\frac{1}{2} \pi \leq \Delta \varphi^{\prime}(x, y) \leq \frac{1}{2} \pi
\end{aligned}
$$




$$
\frac{3}{2} \pi \leq \Delta \varphi^{\prime}(x, y)<2 \pi
$$

which are shown with a slanted line in Fig. 3 as a restriction condition in the reconstructed image of the iterative dummy area method. In this expression, $\Delta \varphi^{\prime}(x, y)$ is $\Delta \varphi(x, y)$ after phase difference restrictions.

We describe the iterative dummy area method. When generating a kinoform which is one type of phase computer-generated holograms, we have to make the amplitude distribution regularity in the hologram distribution. However, an error arises in the reconstructed distribution by forcing the amplitude distribution regularity. An iterative dummy area method has been suggested as an algorithm which reduces this error. The flow of the iterative dummy area method is shown in Fig.5. In the iterative dummy area method, a dummy area with initial distribution zero is added to the surroundings of an original image distribution. The initial distribution after the addition of the dummy area is shown in Fig.6. The reconstruction error can be reduced by using the flexibility of the amplitude and phase in the dummy area. In addition, the size of the dummy area is arbitrary, and can hasten the convergence of the solution by the increasing the size of the dummy area. We carry out iteration by using this initial distribution as an input.

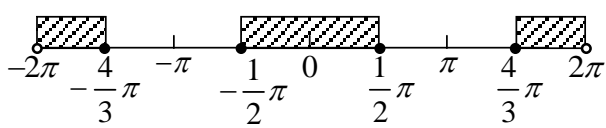

Fig.4. Phase difference constraint
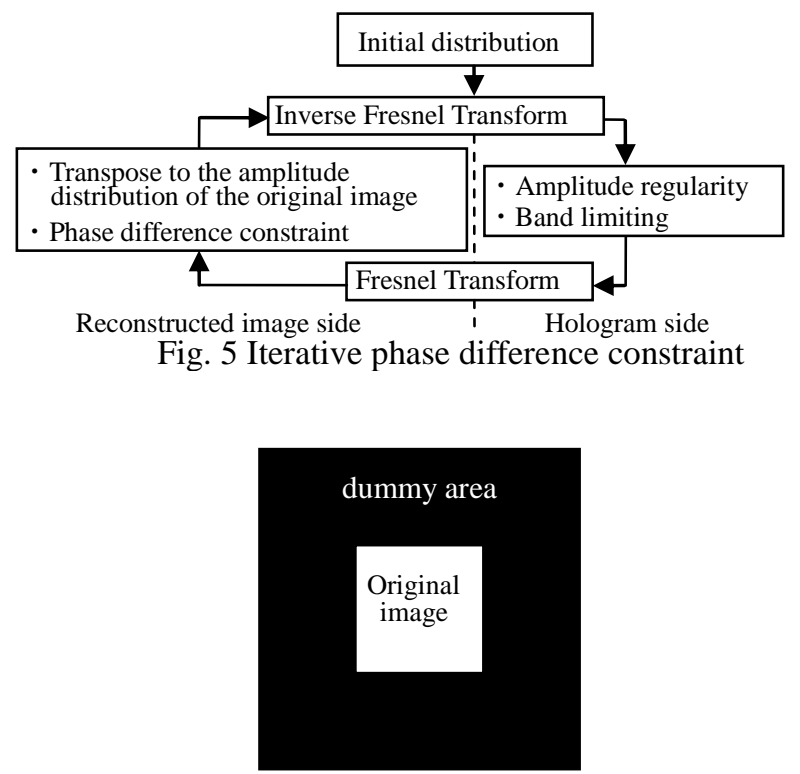

Fig. 6. Dummy area.
Next, we describe the principle of speckle elimination by phase difference constraint method. Case the phase difference is obtained by limiting the range of $\mathrm{K}$ is the following five.

1. The range of Eq. (17) is two, the range of Eq. (19) is two

2. The range of Eq. (18) is four

3. The range of Eq. (17) is one, the range of Eq. (18) is two, the range of Eq. (19) is one

4. $\pi / 2$ is three, $-3 \pi / 2$ is one

$5 .-\pi / 2$ is three, $3 \pi / 2$ is one

Speckle does not occur because it does not satisfy the equation in the case of up to 3 from the top. However, speckle may occur to satisfy the equation in the case of two lower. But, if it becomes such a case, it is possible to eliminate the speckle by exception handling because it can be changed into cases third by adding the $\pi$ or $-\pi$ to appropriate point.

\section{Results}

Simulated is carried out under the following conditions.

$\begin{array}{ll}\text { - Images } & \begin{array}{l}\text { Images with } 64 \times 64 \text { pixel } \\ \text { shown in Fig. } 7\end{array} \\ \text { - Initial phase } & \text { Random phase } \\ \text { - Dummy area } & 256 \times 256[\text { pixel] } \\ \text { - Band limiting } & 1 / 2 \\ \text { - It is repeatedly a frequency } & 5000 \text { times } \\ \text { - Distance } & 1.5[\mathrm{~m}]\end{array}$

The mean square error defined in Eq. (20) is used as the expression of the reconstruction evaluation.

$$
E_{M E S}=10 \cdot \log \frac{\sum \sum|f(x, y)|^{2}-\alpha\left|g_{k}^{\prime}(x, y)\right|^{2}}{\sum \sum|f(x, y)|^{4}}
$$

Fig.8 shows the result in the case of non-despeckle, and FIg.9 shows the result in the case of despeckle. We can see that the speckle is eliminated by the phase difference constraint method in all images. The final reconstructed image errors are $-10.77 \mathrm{~dB}$ in Fig.8(a), -10.04 $\mathrm{dB}$ in Fig.8(b), and $-10.88 \mathrm{~dB}$ in Fig. 8(c), $-37.50 \mathrm{~dB}$ in Fig.9(a), $-30.60 \mathrm{~dB}$ in Fig.9(b), $-33.87 \mathrm{~dB}$ in Fig.9(c), respectively.

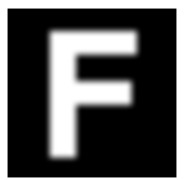

(a) $\lceil\mathrm{F}\rfloor$

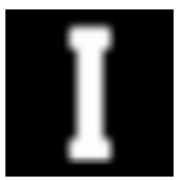

(b) $\lceil\mathrm{I}\rfloor$

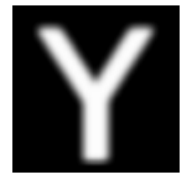

(c) $\lceil\mathrm{Y}\rfloor$
Fig.7. Original image 


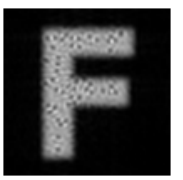

(a) $\lceil\mathrm{F}\rfloor$

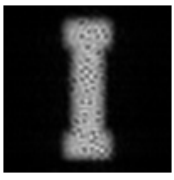

(b) $\lceil\mathrm{I}\rfloor$

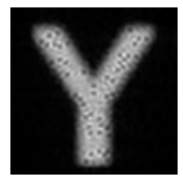

(c) $\lceil\mathrm{Y}\rfloor$
Fig.8. Non-despeckle

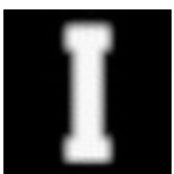

(b) $\lceil\mathrm{I}\lrcorner$

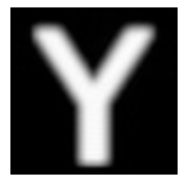

(c) $\lceil\mathrm{Y}\rfloor$ (a) $\lceil\mathrm{F}$

Fig.9. Despeckle

\section{Conclusions}

Speckle noise is a serious problem in the reconstruction of computer-generated holograms (CGHs). Especially for a kinoform (a phase-only CGHs), the speckles lead to a large reconstruction error and it is important to eliminate the speckles. Phase difference constraint method has been proposed as a method for speckle elimination ${ }^{(1)}$. However, simulation of phase difference constraint method is carried out only in the Fourier kinoform. But, considering the optical reconstruction, it preferred to use a Fresnel kinoform. Therefore, we carried out the simulation in Fresnel kinoform.

We carried out the simulation in the three kinds of images "F", "I", "Y", and the speckle elimination has been confirmed in all images. Therefore, the phase difference constraint method is also useful in the Fresnel kinoform was confirmed.

\section{References}

(1) Toshinori Hora, Shiyuan Yang, Seiichi Serikawa :

$\lceil$ Iterative phase difference constraint method for the speckle elimination of kinoforms」,2012

(2) H. Aagedal, M. Schmid, T. Beth, S. Teiwes, and F. Wyrowski, "Theory of speckles in diffractive optics andits application to beam shaping," J. Mod. Opt. No. 43, 1409 - 1421 (1996).

(3) H. Akahori, "Spectrum leveling by an iterative algorithm with a dummy area for synthesizing the kinoform," Appl. Opt., Vol. 25, No. 5, 802-811 (1986).

(4) R.Braer, F.Wyrowski, and O.Bryungdahl, "Diffusers in digital holography”, J.Opt.Soc.Am.A, Vol. 8 No. 3,

572-578(1991)

(5) F. Wyrowsky and O. Bryngdahl, "Speckle-free reconstruction in digital holography," J. Opt. Soc. Am. A, Vol. 6, No. 8, 1171-1170 (1989).

(6) Shiyuan Yang and Hiroaki Takajo, "Speckle reduction of kinoform reconstruction utilizing the $2 \pi$ ambiguity of image phase differences," Optical Review, Vol. 12, No. 2, 93-96 (2005). 